\title{
Molecular markers of the $S K r$ gene in the evaluation of bread wheat genotypes with different crossability with rye
}

\author{
Porotnikov I.V.*, Antonova O.Yu., Mitrofanova O.P. \\ N.I. Vavilov All-Russian Institute of Plant Genetic Resources (VIR), St. Petersburg, Russia \\ *email: i.v.porotnikov@gmail.com
}

Crossability of bread wheat (Triticum aestivum L.) with rye (Secale L.) is controlled by $K r 1-K r 4$ genes and suppressor $S K r$. The recessive alleles cause high crossability with rye, while the dominant ones suppress crossability. The genes $K r l$ and $S K r$ have a major effect on ability to set seed. They were located on 5BL and 5BS respectively. Molecular markers for these genes can force identification of new crossable genotypes and make the increasing of wheat's genetic diversity easier. The purpose of research is to study allelic variation for closely linked to $S K r$ molecular markers and to analyze their association with high crossability with rye. We used 77 crossable and 23 noncrossable genotypes of bread wheat from VIR collection, which were characterized in our experiments or according to literature sources. Every accession was represented by 2-3 genotypes. Three markers from literature: "gene 12", Xcfb341 and Xcfb382 were used for molecular screening. The diagnostic fragment of high crossability for marker "gene 12 " was identified among 90,9\% crossable genotypes, when for Xcfb341 - among $88,3 \%$. However, these fragments were also found in many noncrossable genotypes (43 and $43,5 \%$ respectively). The SSR marker $X c f b 382$ was monomorphic in almost all of genotypes, except for crossable genotypes from k-38587 Sibirka Yartsevskaya (Russia), which had the unique fragment. New alleles that weren't previously described in the literature were also identified at the $X c f b 341$ locus. In total, the six haplotypes were identified based on three markers. The major haplotypes were $\mathrm{H}^{\mathrm{SKr}}-1$ and $\mathrm{H}^{\mathrm{SKr}}-2$, which were found out for 77 and $18 \%$ genotypes respectively. Haplotype $\mathrm{H}^{\mathrm{SKr}}-1$ was revealed the most of crossable genotypes $(85,9 \%)$ and only $56,5 \%$ noncrossable genotypes had haplotype $\mathrm{H}^{\mathrm{SKr}}-2$. Other haplotypes were rare or unique. All of them were found in crossable genotypes. Thus, the use of molecular markers "gene12" and Xcfb341 showed a sufficiently high effectivity for identification of crossable genotypes. The additional markers are needed for more successful searching. 